\title{
Method to Retrieve Bionic Morphologies by Spectral Matching for the Design Space
}

\author{
Rohith Vaidyanathan ${ }^{1,2}$, Gururaj Fattepur ${ }^{2,3}$, Ravi C. Guttal ${ }^{2 *}$ \\ ${ }^{1}$ School of Computer Science, KLE Technological University, Hubballi 580031, India \\ ${ }^{2}$ Center for Innovation and Product Development, KLE Technological University, Hubballi 580031, India \\ ${ }^{3}$ School of Mechanical Engineering, KLE Technological University, Hubballi 580031, India
}

Corresponding Author Email: ravi_g@kletech.ac.in

https://doi.org/10.18280/ijdne.150509

Received: 12 June 2020

Accepted: 16 September 2020

\section{Keywords:}

bionic design, eigen modes, computational geometry, conceptual design, design optimisation, nature-inspired design

\begin{abstract}
Bionic designs have evolved from time-tested strategies of nature and have been a source of inspiration for designers and engineers to solve problems. Nature has a rich repository of sustainable and robust ecosystems evolved since 3.8 million years. Previous research works have shown that bio-inspired structures have demonstrated better performance over conventional design. Thus, over the years, several attempts have been made to systematically derive bionic concepts from the convoluted databases of biological occurrences. The purpose of this research work is to propose an analytical method that can search biological databases to obtain similar forms and aid the conceptual phase of the bionic design process. Eigen modes have proven to be reliable indicators to derive homologous bio-inspired structures. Derivation of bionic structures for the design space is possible after spectral matching of the mode shapes. Inspiration design phase for a bevelled chisel has been demonstrated using this method. A morphological database with form of beaks, domes and wings was leveraged for the case study. The results indicate that the proposed method can enable automatic geometric feature transfer to existing CAD tools. The designer can leverage this analytical method to obtain biological forms from databases and realise novel bionic design concepts.
\end{abstract}

\section{INTRODUCTION}

Bionic designs hold a lot of intrigue and potential because they have been developed by the process of natural selection over millions of years. Nature's forms have inspirations a designer seeks to emulate because of its inherent efficiency or its aesthetics. Mimicking bionic forms can also be viewed as a paradigm for sustainability [1]. Natural selection is driven by mutations which are favorable for survival of the organism in a particular environment. Accumulation and combination of mutations yield great diversity with time. Thus over 10 million species are estimated to exist on Earth [2].

Nature's form is not only exquisite art, it also develops as an adaptation to the environment [3]. Ingo Reichenberg, at the 3rd European Futurists Conference (2007) in Lucerne, stressed that bionic design will drive fundamental rethinking in manufacturing and technical innovations for the next century. The idea of using bionic solutions for structural design was spawned in the early 2000's [4]. Furthermore, bionic inspirations can lead to novel solution spaces waiting to be explored [5]. They augment the design process and provide engineers and designers with an alternate universe of design solutions. The advent of additive manufacturing offers new design possibilities and thus complex yet optimized bionic designs have taken center stage.

Morphological strategies employed by nature have shown impeccable efficiency owing to its evolution over 3.8 billion years [6]. For instance, Bajsanski et al. parameterised a model of the shark skin denticle geometry to design windbreak fences [7]. Simulations comparing shark skin with other geometries revealed that the former substantially reduced wind velocity for all directions. The slime mold, Physarum polycephalum, can connect different sources of food in an efficient manner [8]; this inspired scientists at Airbus to invent a de novo structure for the aircraft cabin partition [9]. The growth process mimicked the network system generated by the slime mold, ultimately obtaining a 50\% lighter structure, not compromising on the state-of-art performance. Similar studies incorporating structural bionics have also reported improvement from traditional structural designs $[10,11]$. Thus, bio-generative designs have evinced novelty for structural design problems. Over the years, functional design methodologies and representation methods with biological strategies have been developed to give engineers and product designers better access to inspirations from nature. However, most methods have been developed to aid creativity in design or modelled functionally which must be searched using keywords. This has restricted the incorporation of biomimetics in industry [12]. Furthermore, the bio-inspired design process is not complete just by choosing the right living prototype; it must fit the given engineering context as well [13]. Project Bionic Aircraft showed how bionics in conjunction with additive manufacturing can be used. Furthermore, it was concluded that existing CAD tools needed to have biomimetic feature recognition for widespread adoption among engineering designers [14].

There is no integrated database which encompasses all biological structures. The vast diversity in species also makes it harder to create a database which will have all the natural structures. Petal by NASA is an initiative of integrating all 
databases into one open source platform [15]. As the data gets bigger, it is hard for the designers to explore nature's concepts. Thus, advanced and faster algorithmic methods are required to search for bionic inspirations. Furthermore, these class of methods must aid in searching vast pools of nature forms quickly.

In this paper, the potential of identifying structural bioinspirations using eigenmodes has been explored. A method to query morphological databases has been proposed by computing and comparing mode shapes. To elucidate the use of this method in the conceptual phase, biological occurrences for a beveled chisel tool has been derived from the bionic form database. The database was constructed using different classes of structures with species names. The scope of the method and its adoption have been discussed.

\section{RELATED WORK}

A survey of literature revealed that bionic design is broadly practiced in three different paradigms. Functional driven analogical design methods are most commonly used in biomimetic design tools. Designers use functional keywords to explore inspirations and try to come up with the design through sketching or 3D modelling. Another group of engineering designer observe the different types of structures across the topology of the nature's occurrence. These structural inferences are used to redesign existing parts and obtain the bionic design. These methods can be categorised as rule based structural bionic design. Some methods make use of geometric databases from which the inspirations are derived. The structures derived are then parametrised and optimised to suit the design problem. These class of methods are of specific interest to this work as they employ morphological retrieval. Inspiration phases of the current methods for bio-inspired design are discussed in the upcoming sub sections.

\subsection{Function driven bionic design}

Previous literature shows that a lot of work has been done for analogical function-driven design processes. This is evident with the developed works of Idea Inspire by Chakrabarti et al. [16] and Design Analogy from Nature (DANE) by Vattam et al. [17]. Both DANE and Idea-Inspire provide qualitative access to information about biological systems. While Idea Inspire is based on the Sapphire analogical model for causality [18], DANE is based on the hierarchical Structure - Behaviour - Function (SBF) model [19]. Ask Nature by Deldin et al. hosts a search engine which stakeholders, irrespective of their background, can use to find biological inspirations [20]; this is based on the Biomimicry taxonomy [21] with over 1600 functional strategy pages. The database is categorised according to taxonomy with the following constructs: Group, Subgroup, Function, and Strategy. The analogical nature of these methods/ models means that most of the data is textual and the search will be primarily through keywords. For instance, to redesign a wheel, the keyword search must include "to move" and "locomotion" which will yield suggestions like "flagella of bacteria" and the "rolling locomotion of the moth caterpillar". Furthermore, it was found that adequate expertise with these models are required to add data to these databases [22].

While function is a reliable way to categorise and search biomimetic designs, search using geometric features can give rise to additional design solutions not obtained functionally. For instance, bats use mammalian limb traits as wings and whales use them as fins. However, the underlying structures are similar despite change in bone sizes [23]. The limbs of all these organisms have evolved from a common ancestor affirming their homology. Thus, a function-only approach can omit designs which do not fall under a particular functional category. Furthermore, convoluted data about biological structures and its systems add to more effort by designers in the inspiration phase of the product development process [24]. An algorithm which can suggest bionic forms to the designer will aid the conceptual design phase and the designers can focus more on the validation phase.

\subsection{Rule-based structural bionic design}

Rule-based structural bionics have led to substantial improvements in structural design process. Generally, the inspiration to the conventional structure stems from similar and heuristic rules in nature. These are transferred to the context of the design space leading to optimised bio-inspired structures. For instance, arrangement of structures in many biological occurrences are such that they display high strength with lower weight. Taking inspiration from the high surface to volume ratio of the plate morphology of a leaf, Xing et al. designed a high-speed machine tool working table and selected rules based on statistical analysis [25]. Distribution of rules in the leaf were studied by statistically analysing leaves from 100 species of plants. Gao et al. redesigned a machine tool column using the stiffness rules by studying leaf veins [10]. The mid veins play a vital role in maintaining global stiffness and the lateral veins add the local stiffness. Zhao et al. also stiffened the ribs inside the machine tool using structural bionics of bones and sandwich stems [26]. In all these studies, the biological structures were selected to replace the rib structures in the machine tool column and the working table based on the deformation values from the finite element method. The veins of the leaf were emulated to optimise the local and global stiffness of the design space.

Some methods of structural bionics rely on redesigning the structure or space using the rules derived from interesting behaviour observed in nature. Nagy et al. developed a computational geometry system which searches for novel design using generative design [9]. As slime mould grows, it first spreads out a dense network of connections. Then, based on where it finds food, it starts to prune the network to keep only those connections that most efficiently connect the food sources. This behaviour forms complex adaptive networks that are efficient and redundant. Here the strategy of the slime mould was selected on the hypothesis that the complex and efficient food distribution networks formed by the slime mould could be beneficial in structural design. The strategy leads to a bracket structure with reduced material. However, heuristic inspirations have no particular input and other forms of networks in the solution pool could have been explored. Derived rules were also parametrized and optimized to find a suitable solution to the design space. In the work by Ivana et al., shark skin denticle geometry was chosen for designing windbreak fences because most drag reducing surfaces mimic this geometry [7]. The morphology was later parametrized to a generative model to estimate improvements according to the design problem. 
Table 1. Comparison of conceptual phase capabilities of the various bionic design processes

\begin{tabular}{cccc}
\hline Method to obtain inspiration & \multicolumn{2}{c}{$\begin{array}{c}\text { Search } \\
\text { Keywhod available } \\
\text { Geometric feature based }\end{array}$} & References \\
\hline Idea-Inspire (Chakrabarti et al.) & $\checkmark$ & $\mathbf{X}$ & {$[16]$} \\
DANE (Vattam et al.) & $\checkmark$ & $\mathbf{X}$ & {$[17]$} \\
Ask Nature (Deldin et al.) & $\checkmark$ & $\mathbf{X}$ & {$[20]$} \\
Bio-Triz (Bogatyrev et al.) & $\times$ & $\mathbf{X}$ & {$[27]$} \\
E2B Thesaurus (McInerney et al.) & $\checkmark$ & $\mathbf{X}$ & {$[28]$} \\
Biocards (Lenau et al.) & $\checkmark$ & $\mathbf{X}$ & {$[29]$} \\
Bionic Design Catalogue ( Lachmayer et al.) & $\checkmark$ & $\mathbf{X}$ & {$[30]$} \\
An agent-based method (Parras et al.) & $\times$ & $\mathbf{X}$ & {$[31]$} \\
Elise 3D (Maier et al.) & $\mathbf{X}$ & $\checkmark$ & {$[32]$} \\
\hline
\end{tabular}

Bionic Design using rules of the nature's geometry have certainly led to better structural characteristics. However, the lack of a method to suggest structural inspirations led to a design process which was guided by one particular organism and occurrences with similar forms were omitted.

\subsection{Bionic design methods employing morphology retrieval}

A considerable amount of work has been done in searching biological occurrences using functional models and heuristic searches. However, designers face the problem of acquiring initial geometries and explore vast amount of similar form inspirations. Hence, morphological databases and methods to acquire data can help designers derive the geometry of the design space; they can focus more on validating the bionic designs acquired.

In order to exploit the advantages of additive manufacturing and the tool-less approach of selective laser melting printers, Lipper et al. manufactured complex bio-inspired lattice geometries [30]. The various bionic occurrences were classified into its respective forms as Bionic Design Elements (BDE). The BDE's were later organised as design catalogues. These bio-inspired structures were mapped to the design space based on the suitability of the load case.

Utilising a database of $1,00,000$ species and their light weight structure geometries, Hamm et al. developed an engineering tool, Elise 3D, to solve this problem. It draws on the shell design of high-strength, unicellular marine organisms such as diatoms and radiolaria to provide design concepts for large scale technical lightweight structures [32]. The lookup from the database was performed by determining the nearest neighbours from a multidimensional scaling map. The functional coordinate points of the design space are needed as input from the designer to find a suitable inspiration.

Table 1 which reviews inspiration phase capabilities by well-known bionic design methods reveal that most methods perform functional search and only a handful focus on biomimetic forms. Designers need to model the design from scratch with the information obtained from a textual search of bionic inspirations. Searching using bionic morphologies easily fits the technical workflow of engineering designers and eliminates the need to search for initial models. In this case, the initial model is directly obtained from the database. As discussed in the previous sections, there is a need for biomimicking techniques which can mimic bionic forms and shapes on the basis of unique structural characteristics. This characteristic must be independent of scale (size) and must hold good for vast ranges of bionic forms. The upcoming section proposes the methodology.

\section{METHODOLOGY}

\subsection{Modal deformations to lookup bionic structures}

Structural forms in nature must be characterized in an analogous fashion to the engineering design space. Hence, bionic structures similar in the macro form need to be suggested to the designer. The relation must capture the salient and significant form features of the structure. In nature, corrugations and complex network of venations (Figure 1), observed in shells, leaves, stems and other bionic parts have been found to yield enhanced mechanical strength. For effective characterization, these intricate and complex structures found in bionic designs must not affect the design representation.
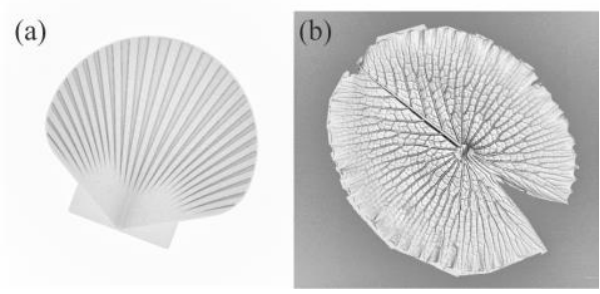

Figure 1. Intricate structures in bionic occurrences (a) Ridges in a shell (b) Venations in a water lily leaf

Harry Blum proposed the Medial Axis Transform (MAT) to characterize biological shapes [33]. However, MAT transform is very unstable to boundary noise. Spurious branches can be formed and additional pruning must be done to obtain an accurate representation. The general use of eigen representations involves selecting important principal components and performing decompositions. These are useful for showing correspondences in shape but they do not depict structural behaviour [34]. Modal analysis shows the limits of a system's response (displacement) for a load of certain amplitude and frequency. Mechanical systems can be formulated in terms of a stiffness matrix $\mathrm{K}$ connecting the forces and the displacements. The eigen vectors $\varphi$ of $\mathrm{K}$ are characteristic vectors of the basis which only vary by scalar values and hence are indifferent to transformations. The eigen basis (or eigen vector basis) covers all the deformations of the structure by way of linear combination and they form an orthogonal basis which is unique to that structure. Thus, eigenmodes and their deformations act as unique signatures of the structure. Firstly, the equilibrium problem is formulated in the form of its elastic stiffness matrix. Finite element method is used to solve this equation. The dynamic equilibrium equation is: 


$$
M \ddot{U}+D \dot{U}+K U=R
$$

where, $\mathrm{K}$ is stiffness tensor, $\mathrm{M}$ is mass tensor, $\mathrm{D}$ is the damping matrix and $\mathrm{R}$ is the load vector. Hence, the mass and stiffness matrices can be derived directly,

$$
M=\int_{V} \rho H^{T} H d V \text { and } K=\int_{V} B^{T} C B d V
$$

where, $\mathrm{H}$ is the element interpolation matrix, $\mathrm{B}$ is the strain displacement matrix, rho is mass density and $\mathrm{C}$ is constitutive material matrix. The mass and stiffness tensors from Eq. (2) are substituted in Eq. (1). Eigen values become complex numbers if damping is considered. Therefore, to reduce computational effort and complexity, damping is not considered in (1). The equation is solved by the Block Lancos method. The mode shapes and frequencies are obtained by the third invariant given by Eq. (3),

$$
\operatorname{det}\left([K]-\omega_{i}^{2}[M]\right)=0
$$

At discrete eigenvalues ( $\omega$ i), the third invariant (det) becomes zero. Hence, the eigenvector( $\varphi$ i) derived from Eq. (4),

$$
\left[K-\omega_{i}^{2} M\right]\left\{\varphi_{i}\right\}=0
$$

The eigenmodes obtained are arranged in the increasing order of frequency. Modes of lower frequencies are influenced by global features whereas the modes of higher order are governed by intricate features. Most of the time it is observed that conventional designs are close to what nature designs. However, due to evolution and the 3.8 billion years spent by biological occurrences, its designs tend to be complex and more focused on intricate local designs (stress and weight concentrated regions) as discussed in the previous section.

Thus, to effectively derive from morphological databases, mode shapes that describe global features (i.e. lower frequency) are important. Furthermore, low order modes require less computation which is an added advantage to go through big databases. Hence, Eigenmodes are chosen to characterize bionic forms and the design space.

\subsection{Comparison of the eigen modes}

The procedure uses discretised models from the database. The discretisation must capture the intricacies of the bionic form used for comparison. As formulated in the previous section, the eigen modes of the design space in consideration are computed from the database. After extraction of the modes, the deformations must be normalised by scale. The Hausdorff distance (a metric for geometric matching) between the fundamental modes of the bionic form and the design space will determine whether the bionic form can be suggested to the designer or not.

The mode shapes of the design space are converted to a point cloud and compared to the corresponding mode shapes of the bionic forms. Scale invariance is a factor that must be considered when bionic forms need to be mimicked [35]. Therefore, the mode shapes are converted to the same scale by determining the maximum bounding box dimension. Iterative closest point (ICP) method is used to align the models in comparison. A transformation is applied to the bionic structure with the design space as a reference. The alignment is done ensuring maximum overlap. Cloud to Mesh (C2M) distance algorithm is used to compare the mode shapes. The orthogonal distance from the point in the cloud to the nearest triangular element is computed. If the orthogonal distance, does not fall in the triangle, the shortest distance to one of the edges of the triangle is taken [36]. The mean of the absolute distances $\left(\mathrm{d}_{\mathrm{m}}\right)$ is the similarity index after comparison given by,

$$
d_{m}\left(S, S^{\prime}\right)=\frac{1}{|S|} \int_{s} d\left(p, S^{\prime}\right) d s
$$

where, $\mathrm{S}$ and $\mathrm{S}$ ' are the two geometries in consideration, $\mathrm{p}$ is the point on S. A prerequisite for this method is a database of bionic morphologies. In the implementation, a database has been constructed for the purpose of this work. However, designers can use this method to query existing structural databases.

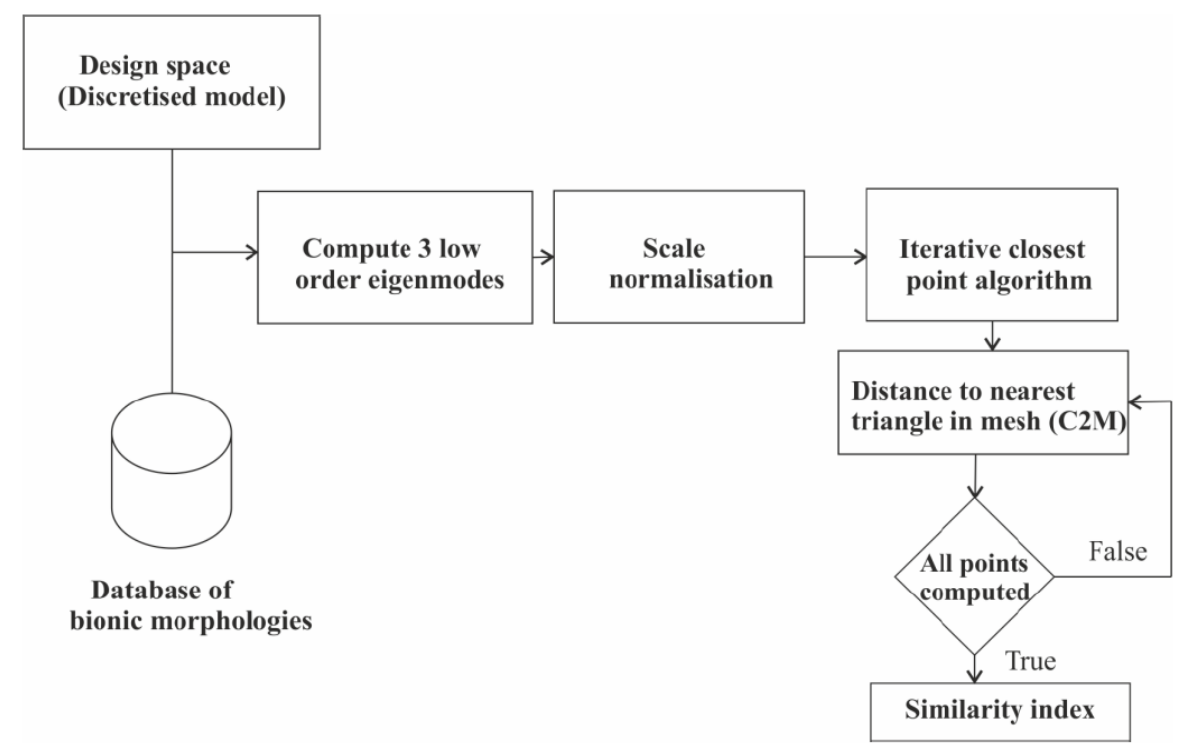

Figure 2. Methodology to derive bionic forms from morphological databases 


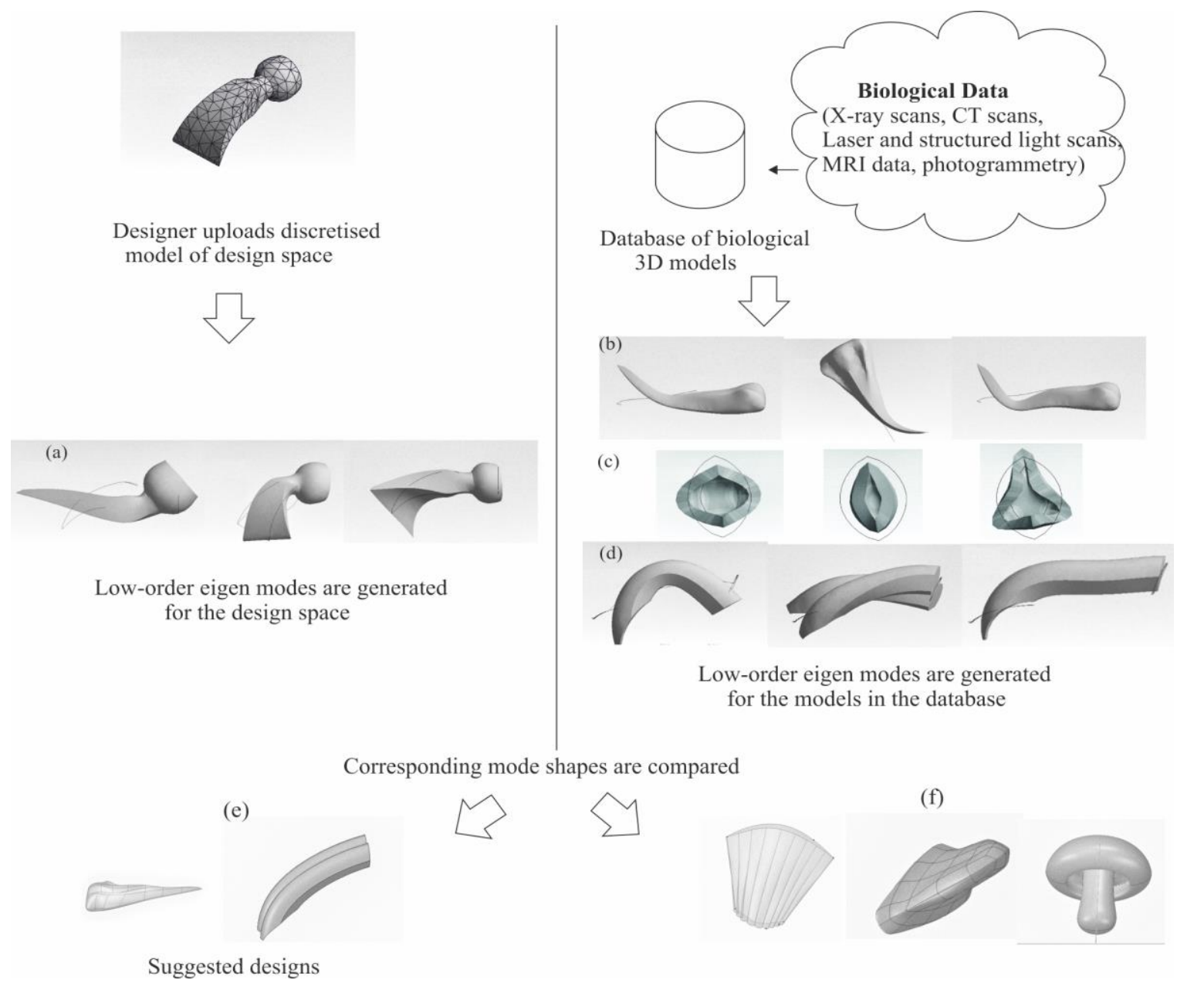

Figure 3. Overview of the proposed approach leveraged for the case study. The three generated eigenmodes of the (a) Design space - bevelled chisel (b) Woodpecker's beak (c) Mushroom crown (d) Mole rat's teeth (e) Designs like beaks and teeth suggested after comparison (f) Forms like Shells, Duck’s beak, Mushrooms not suggested to designer

\section{CASE STUDY - BIONIC FORM TO A DESIGN SPACE}

The objective is to find the inspirations for the discretized model uploaded by the designer using the proposed paradigm. For this work, a database was formed using a combination of bionic structures (wings, beaks, crowns, teeth) from different biological species taking key aspect ratios as reference. Using the database created for this work, the proposed method in Figure 2 is leveraged to find a suitable inspiration. Cloud Compare has been used for 3D processing [37].

\subsection{Inspiration phase for a hand tool}

A beveled chisel was chosen as the case for which bionic inspirations were derived using the proposed method as illustrated in Figure 3. The width of the chisel is $121 \mathrm{~mm}$. A discretized model of the chisel must be uploaded by the designer. Low-order eigen modes are generated for the design space and a model from the morphological database. The mode shapes are iteratively generated for the beaks, domes and wings in the database. The modal deformations are represented in Figure 3 (a), (b), (c) and (d). Once, the modal analysis was performed corresponding mode shapes are compared with the modes of the chisel generated in the previous step.

To perform cloud to mesh comparison, points are sampled on the design space to convert it to a point cloud. Thus, distance comparison is done for the design space with all the category of models in the database (Figure 4).

Upon comparison, the closest bionic designs are suggested. The mean of absolute distance values is tabulated in Table 2 .

Table 2. Mean distances of beveled chisel (design space) with other bionic morphologies

\begin{tabular}{ccccc}
\hline & & \multicolumn{3}{c}{$\begin{array}{c}\text { Mean distance from absolute } \\
\text { distances (mm) }\end{array}$} \\
\cline { 3 - 5 } No. & Case & $\mathbf{1}^{\text {st }}$ mode & $\mathbf{2}^{\text {nd }}$ mode & $\mathbf{3}^{\text {rd }}$ mode \\
\hline 1$)$ & Woodpeckers beak & 19.392 & 10.956 & 13.871 \\
$2)$ & Flammulina & 93.72 & 41.173 & 98.564 \\
& velutipes crown & & & \\
$3)$ & Mole rat teeth & 12.314 & 11.007 & 15.212 \\
$4)$ & Bat wings & 60.651 & 45.321 & 70.834 \\
$5)$ & Agaricus bisporus & 75.212 & 52.936 & 79.716 \\
\hline
\end{tabular}

Figure 4. Registration and Cloud to Mesh comparison from the point cloud of woodpecker's beak to the bevelled chisel (design space) 


\subsection{Discussion}

The case study illustrated shows the working of the proposed method in this work. The mole rat's teeth and the woodpecker's beak are evidently homologous and are suggested as inspirations to the design space using the proposed method. Figure 6 shows the distance maps of the bionic structure with the case study (beveled chisel). The results show that similar form structures have similar eigenmodes even though bionic morphologies have intricate changes in structure due to evolution. This can lead to optimal designs which have a higher strength to weight ratio. Since, the mode shapes depict structural behavior it is also interesting to see the correlations between different eigenmodes with the design space. From the table, we can see that the $1^{\text {st }}$ eigenmode of the teeth of a mole rat is closer than the woodpecker's beak. However, the second and third mode shapes of the woodpecker's beak is closer to the beveled chisel. Structures of Flammulina velutipes and Agaricus bisporus mushrooms showed a very high distance metric as compared to the other forms. This is because the first three eigenmodes show no resemblance to that of the tool. Figure 5 (a), (b) and (c) show the histograms of the absolute distances between the three fundamental modes of the beak to the design space (beveled chisel). Similarly, Figure 5 (d), (e) and (f) are histograms plotted for the mushroom's crown to the design space. The change in modal distances can be observed from the graphs. After conceptual inspirations are obtained using this algorithm, the designer can validate his design using engineering simulations.
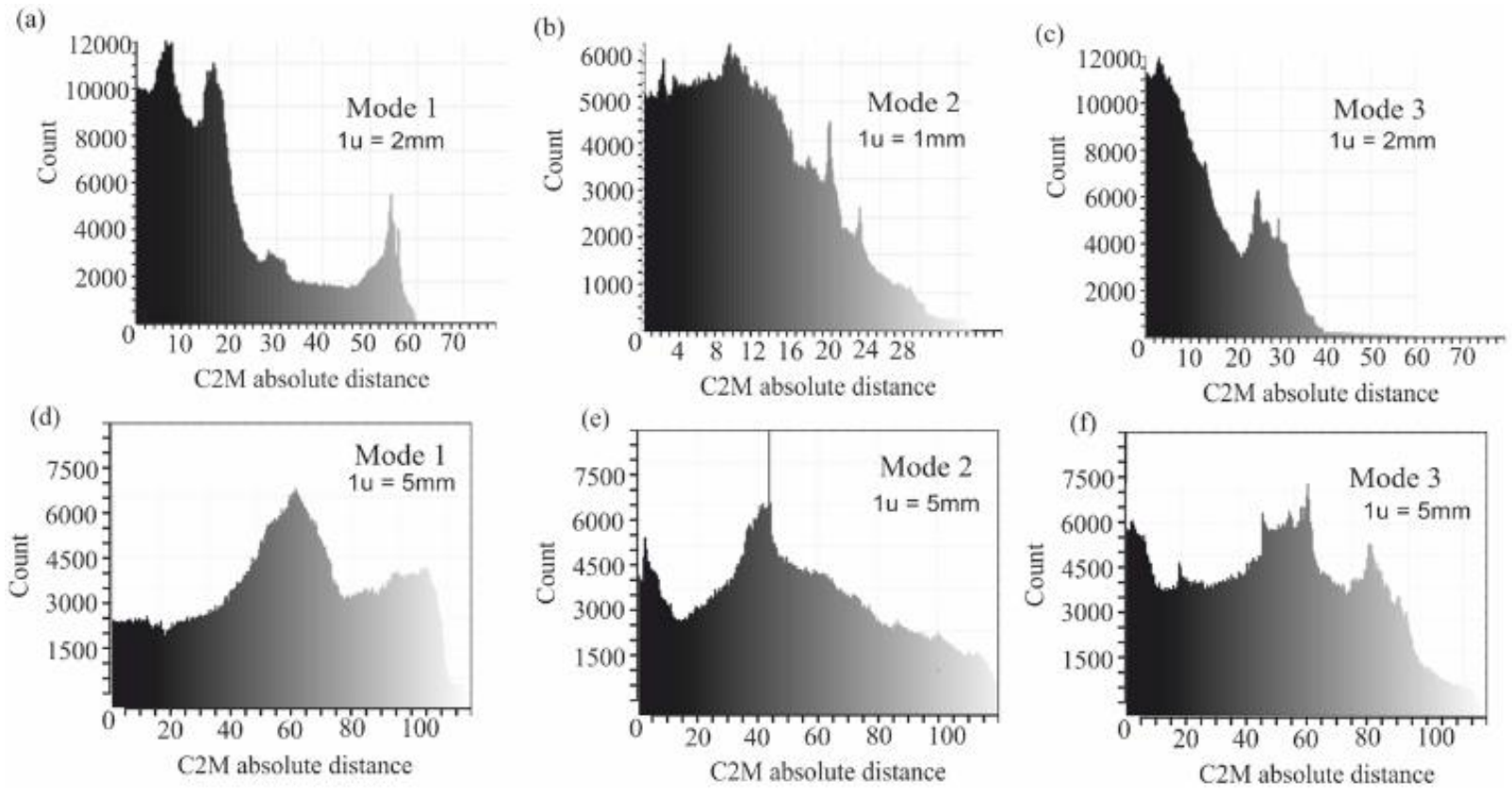

Figure 5. (a), (b), (c) Histograms of absolute comparison distances of the design space under consideration with woodpecker's beak (d), (e), (f) with the mushroom's crown
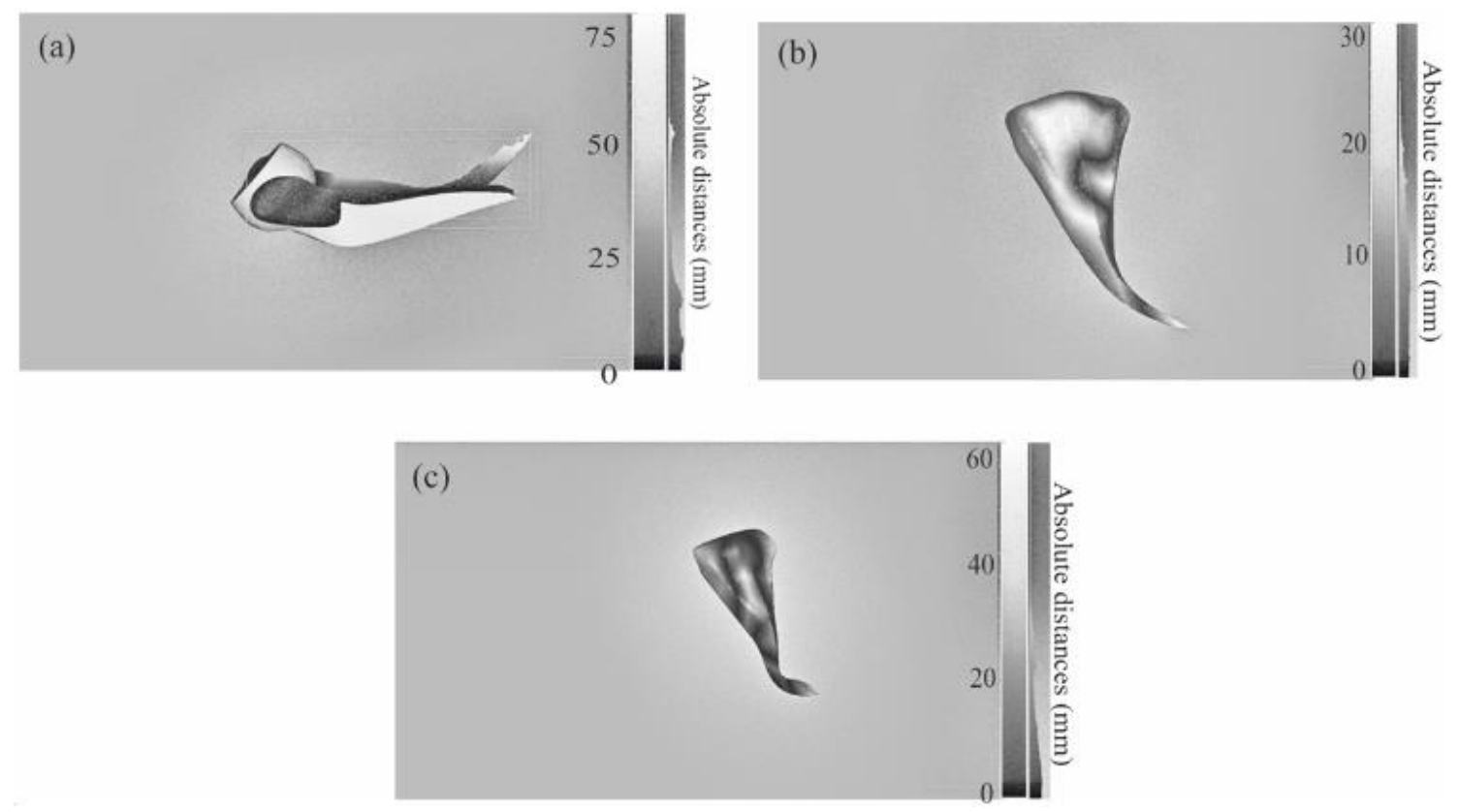

Figure 6. Distance maps which illustrate the absolute distances of the eigenmodes of design space (bevelled chisel) with the woodpecker's beak (a) First eigenmode (b) Second eigenmode (c) Third eigenmode 


\subsubsection{Advantages}

(1) The method proposed in this work is suited for designers and engineers who are looking for form concepts similar to the design space. These bionic structures could have better dimensional ratios which could lead to better structural characteristics.

(2) This methodology can also be integrated with current CAD tools to query databases with bionic morphologies.

(3) The method is scale invariant due to normalisation of the fundamental modes.

\subsubsection{Limitations}

(1) The proposed method can only be used for finding bionic structures which are similar in form. However, forms like a complex network of roots cannot be mimicked effectively with the proposed method. The modes can show erroneous deformations and thus comparison becomes a difficulty.

(2) This method works well if there is a sufficiently large centralised database of bionic design models with different types of structures. However, one drawback is that such a database comprising of all-natural structures is not present. Models are staggered across different datasets.

(3) The morphologies used in this study were already segmented. A robust segmentation strategy which can be applied to derive constituent parts from the model of a whole organism could be a future addition to this method.

\section{CONCLUSION AND FUTURE SCOPE}

Structures of nature exhibit tremendous complexity and autonomy in form. These designs also show multifunctionality; a trait which designers seek to incorporate in current structures. Bionic Design is opening new avenues to practice structural design. Engineering industries are embracing the idea of looking into nature for light-weight, robust and novel solutions. Furthermore, previous studies have shown that utilizing rules from nature to engineering designs can lead to resilient structures.

Thus, designers require methods that can search for vast inspirations found in nature. Despite prior research on keyword-based retrieval mechanisms, only a few studies have focused on retrieving bionic morphologies. Thus, in this work, an algorithm is proposed to derive bio-inspired structural inspirations for the design space. It employs computation of low-order eigen modes which capture global features of the bionic models for effective biomimetic transfer to the design space.

This method of characterizing nature using global encodings involving principal components can be extended to other domains like mechanisms, energy and materials. Lattice structures/ microstructures have also been found to distribute loads effectively with a high strength to weight ratio. Methods to characterize and retrieve microstructures can also be used in conjunction with this work.

Future scope will also involve development of technical problem-solving design methodologies which incorporates the complete product development lifecycle. Such a product lifecycle method can lead to novel tools which can give better access to natural occurrences for designers who are not necessarily from a biological background. Thus, increased adoption of nature's strategies can ensure a sustainable future.

\section{REFERENCES}

[1] De Pauw, I., Kandachar, P., Karana, E., Peck, D., Wever, R. (2010). Nature inspired design: Strategies towards sustainability. Knowledge Collaboration \& Learning for Sustainable Innovation, 1: 1-21.

[2] Ayala, F.J. (2007). Darwin's greatest discovery: Design without designer. Proceedings of the National Academy of Sciences of the United States of America, 104(1): 8567-8573. https://doi.org/10.1073/pnas.0701072104

[3] Yin, J., Yang, W.C. (2017). Review of the research on "structural bionic" method of large sculpture. IOP Conference Series: Materials Science and Engineering, 242: $\quad 012083 . \quad$ https://doi.org/10.1088/1757899X/242/1/012083

[4] Murawski, K., Arciszewski, T., De Jong, K. (2000). Evolutionary computation in structural design. Engineering with Computers, 16: 275-286. https://doi.org/10.1007/PL00013716

[5] Gawell, E., Nowak, A., Rokicki, W. (2019). Searching for bionics structural forms optimization. IOP Conference Series: Materials Science and Engineering, 471(5): $\quad$ 052066. $\quad$ https://doi.org/10.1088/1757899X/471/5/052066

[6] Benyus, J. (2002). Biomimicry: Innovation Inspired by Nature. Harper Perennial.

[7] Bajsanski, I., Stojaković, V., Tepavcevic, B., Jovanović, M., Mitov, D. (2017). An application of the shark skin denticle geometry for windbreak fence design and fabrication. Journal of Bionic Engineering, 14: 579-587. https://doi.org/10.1016/S1672-6529(16)60423-7

[8] Tero, A., Takagi, S., Saigusa, T., Ito, K., Bebber, D., Fricker, M., Yumiki, K., Kobayashi, R., Nakagaki, T. (2010). Rules for biologically inspired adaptive network design. Science, 327(5964): 439-442. https://doi.org/10.1126/science.1177894

[9] Nagy, D., Zhao, D., Benjamin, D. (2018). Nature-based hybrid computational geometry system for optimizing component structure. In: De Rycke K. et al. (eds) Humanizing Digital Reality. Springer, Singapore. https://doi.org/10.1007/978-981-10-6611-5_15

[10] Gao, H.J., Sun, J.F., Chen, W.Y., Zhang, Y.D., Wu, Q. (2018). Structural bionic design for a machine tool column based on leaf veins. Proceedings of the Institution of Mechanical Engineers, Part C: Journal of Mechanical Engineering Science, 232(16): 2764-2773. https://doi.org/10.1177/0954406217726565

[11] Jiao, H.J., Zhang, Y.D., Chen, W.Y. (2010). The lightweight design of low RCS pylon based on structural bionics. Journal of Bionic Engineering, 7: 182-190. https://doi.org/10.1016/S1672-6529(09)60207-9

[12] Vincent, J. (2020). Why don't more commercial companies take up biomimetics? International Society of Bionic Engineering, 9(1): 11

[13] Coelho, D., Versos, C.A.M. (2011). A comparative analysis of six bionic design methods. International Journal of Design Engineering, 4(2): 114-131.

[14] Airbus Newsletter. (2019). Bionic Toolset. https://bionicaircraft.eu/wpcontent/uploads/2019/09/Newsletter_Aug ust_2019.pdf, accessed on 15 Sep. 2020.

[15] https://github.com/nasa/PeTaL, accessed on 15 Sep. 2020.

[16] Chakrabarti, A., Siddharth, L., Dinakar, M., Panda, M., 
Palegar, N., Keshwani, S. (2017). Idea inspire 3.0-A tool for analogical design. International Conference on Research into Design, Research into Design for Communities, 2: 475-485. https://doi.org/10.1007/978981-10-3521-0_41

[17] Vattam, S., Wiltgen, B., Helms, M., Goel, A., Yen, J. (2011). DANE: Fostering creativity in and through biologically inspired design. In: Taura T., Nagai Y. (eds) Design Creativity 2010. Springer, London. https://doi.org/10.1007/978-0-85729-224-7_16

[18] Chakrabarti, A., Sarkar, P., Leelavathamma, B., Nataraju, B.S. (2005). A functional representation for aiding biomimetic and artificial inspiration of new ideas. AIE EDAM, 19(2): 113-132. https://doi.org/10.1017/S0890060405050109

[19] Goel, A., Rugaber, S., Vattam, S. (2009). Structure, behavior, and function of complex systems: The structure, behavior, and function modeling language. AI EDAM, 23(1):

$23-35$. https://doi.org/10.1017/S0890060409000080

[20] Deldin, J.M., Schuknecht, M. (2014). The AskNature database: Enabling solutions in biomimetic design. In: Goel A., McAdams D., Stone R. (eds) Biologically Inspired Design. Springer, London. https://doi.org/10.1007/978-1-4471-5248-4_2

[21] THE Biomimicry Taxonomy: Biology Organized by Function. http://toolbox.biomimicry.org/wpcontent/uploads/2015/01/AN_Biomimicry_Taxonomy.p df, accessed on 15 Sep. 2020.

[22] Siddharth, L., Chakrabarti, A. (2018). Evaluating the impact of Idea-Inspire 4.0 on analogical transfer of concepts. Artificial Intelligence for Engineering Design, Analysis and Manufacturing, 32(4): 431-448. https://doi.org/10.1017/S0890060418000136

[23] Lin, Q., Fan, S.H., Zhang, Y.H., Xu, M., Zhang, H.X., Yang, Y.L., Lee, A., Woltering, J., Vydianathan, R., Gunter, H., Luo, W., Gao, Z.X., Lim, Z.W., Qin, G., Schneider, R., Wang, X., Xiong, P.W., Li, G., Wang, K., Min, J.M., Zhang, C., Qiu, Y., Bai, J., He, W.M., Bian, C., Zhang, X.H., Shan, D., Qu, H.Y., Sun, Y., Gao, Q., Huang, L.M., Shi, Q., Meyer, A., Venkatesh, B. (2016). The seahorse genome and the evolution of its specialized morphology. Nature, 540: 395-399. https://doi.org/10.1038/nature20595

[24] Shyam, V., Friend, L., Whiteaker, B., Bense, N., Dowdall, J., Boktor, B., Johny, M., Reyes, I., Naser, A., Sakhamuri, N., Kravets, V., Calvin, A., Gabus, K., Goodman, D., Schilling, H., Robinson, C., Reid II, R.O., Unsworth, C. (2019). PeTaL (Periodic Table of Life) and physiomimetics. $\quad$ Designs, 3(3): 43. https://doi.org/10.3390/designs3030043

[25] Xing, D.H., Chen, W.Y., Zhao, L., Ma, J.F. (2012). Structural bionic design for high-speed machine tool working table based on distribution rules of leaf veins. Science China Technological Sciences, 55: 2091-2098.

[26] Zhao, L., Chen, W.Y., Ma, J.F., Yang, Y.B. (2008). Structural bionic design and experimental verification of a machine tool column. Journal of Bionic Engineering, 5: 46-52. https://doi.org/10.1016/S1672-6529(08)60071-2

[27] Bogatyrev, N., Bogatyreva, O. (2015). TRIZ-based algorithm for biomimetic design. Procedia Engineering, 131: 377-387.

[28] McInerney, S., Khakipoor, B., Garner, A., Houette, T.,

Unsworth, C., Rupp, A., Weiner, N., Vincent, J., Nagel, J., Niewiarowski, P. (2018). E2BMO: Facilitating user interaction with a biomimetic ontology via semantic translation and interface design. Designs, 2(4): 53. https://doi.org/10.3390/designs2040053

[29] Lenau, T.A., Keshwani, S., Chakrabarti, A., AhmedKristensen, S. (2015). Biocards and level of abstraction. ICED 15 - International Conference on Engineering Design at: Politecnico Di Milano, Italy, 2: 1-10.

[30] Lippert, R.B., Lachmayer, R. (2017). A design method for SLM-Parts using internal structures in an extended design space. International Conference on Additive Manufacturing in Products and Applications, pp. 14-23. https://doi.org/10.1007/978-3-319-66866-6_2

[31] Parras, D., Fernández-Pacheco, D., Cavas-Martínez, F., Martínez, J.N., Canavate, F. (2018). An agent-based approach for the application of nature's forms to product conceptual design. PLOS ONE, 13: e0208930.

[32] Maier, M., Hamm, C. (2011). ELISE 3D - A databasedriven engineering and design tool. ICED 11 - 18th International Conference on Engineering Design Impacting Society Through Engineering Design, 9: 132142.

[33] Blum, H. (1973). Biological shape and visual science (part I). Journal of Theoretical Biology, 38(2): 205-287.

[34] Sclaroff, S., Pentland, A.P. (1995). Modal matching for correspondence and recognition. IEEE Transactions on Pattern Analysis and Machine Intelligence, 17(6): 545561. https://doi.org/10.1109/34.387502

[35] Kazhdan, M. (2004). Shape Representations and Algorithms for 3D Model Retrieval. PhD Thesis, Princeton University. https://gfx.cs.princeton.edu/pubs/_2004_SRA/.

[36] Jones, M. (1995). 3D Distance from a Point to a Triangle. Technical Report, Department of Computer Science, University of Wales Swansea. http://cs.swan.ac.uk/ csmark/PDFS/1995_3D_distance_ point_to_triangle, accessed on 15 Sep. 2020.

[37] CloudCompare (version 2.11.0) [GPL software]. (2020). Retrieved from http://www.cloudcompare.org, accessed on 15 Sep. 2020.

\section{NOMENCLATURE}

K
M
D
R
H
B
C

\section{Greek symbols}

$\rho$

$\omega$

$\varphi$

\section{Subscripts}

det stiffness tensor,_N/m

mass tensor, $\mathrm{kg}$

dimensionless damping coefficient

load vector, $\mathrm{N}$

element interpolation matrix

strain displacement matrix, $\mathrm{m}$ material constitutive matrix, $\mathrm{N} / \mathrm{m}^{2}$

mass density, $\mathrm{kg} \cdot \mathrm{m}^{-3}$

eigen vector, $\mathrm{m}$

eigen values, $\mathrm{s}^{-1}$

determinant 